\title{
Neutrophil Albumin Ratio is Associated with All-Cause Mortality in Stroke Patients: A Retrospective Database Study
}

\author{
Zhibo Chen' \\ Dewei Xie' \\ Yun $\mathrm{Li}^{1}$ \\ Zebin $\mathrm{Dai}^{2}$ \\ Saina Xiang ${ }^{2}$ \\ Zhiyuan Chen ${ }^{2}$ \\ Weiqian Zhu'
}

'Department of Neurology, The First Affiliated Hospital of Wenzhou Medical University, Wenzhou, Zhejiang Province, People's Republic of China; ${ }^{2}$ College of the First Clinical Medical Sciences of Wenzhou Medical University, Wenzhou, Zhejiang, People's Republic of China
Correspondence: Weiqian Zhu Department of Neurology, The First Affiliated Hospital of Wenzhou Medical University, Wenzhou, 325000, Zhejiang Province, People's Republic of China

Tel +8657755579372

Fax +8657755579318

Email zhuwqfyxy@aliyun.com
Objective: The novel biomarker, neutrophil percentage-to-albumin ratio (NPAR), as a prognostic tool for inflammation in relation to all-cause mortality for patients afflicted by strokes has yet to be explored.

Methods: Data sets associated with patient files stored within the MIMIC-III V1.4 database were obtained. Data files from 940-patients were obtained for this retrospective analysis. Clinical endpoints were determined to represent a month (30-), three months (90-) and year (365-) all-cause mortality in stroke patients were determined. In order to determine NPAR and clinical endpoint relationships, Cox proportional hazards models were utilized.

Results: For all-cause mortality within a 30-day period, in an unadjusted model, the HR (95\% CIs) in group B (NPAR 20.5-25.0) and C (NPAR >25.0) was $1.17(0.85,1.63)$ and $1.55(1.13,2.11)$ compared with group A (NPAR < 20.5). Proceeding adjustment for more confounding factors, higher NPAR still obtained significant predictive power for 30-day allcause mortality $(\mathrm{HR}=1.45,95 \% \mathrm{CI}: 1.05,2.00)$. Statistical significance $(P=0.0196)$ was also observed for the other time-based subgroupings for all-cause mortality.

Conclusion: A strong correlation was present between increased levels of the novel biomarker NPAR and increased risk of mortality in stroke patients.

Keywords: neutrophil-albumin ratio, mortality, stroke, biomarker

\section{Introduction}

Stroke is reported to be the leading lethal disease in People's Republic of China, which is also the principal cause of disability among adults. ${ }^{1,2}$ Ischemic stroke accounts for $80 \%$ of all kinds of stroke. ${ }^{3}$ Stroke may affect the first stroke patients again at a specific time point after rehabilitation in hospital. ${ }^{4}$ Among patients in intensive care unit, critical stroke is very common. ${ }^{5}$ However, the treatment outcomes are not satisfied for stroke patients, which brings their family and society great burden and economic loss. ${ }^{3}$ Therefore, it is urgent to find a predicting biomarker of the prognosis of patients who are in an early stage of stroke.

The pathogenesis of stroke is closely related to inflammation, and the pathological processes involved in the development of acute ischemic stroke include endothelial activation, blood-brain barrier impairment, secretion of multiple inflammatory mediators, oxidants, and cytokines, and infiltration of platelet and leukocytes. ${ }^{6,7}$ 
NPAR (neutrophil percentage-to-albumin ratio) was a newly reported inflammatory biomarker. ${ }^{8}$ The prognostic and predictive functions of NPAR were found in various diseases, such as cardiovascular disease. ${ }^{9}$ It was reported that reduced Albumin levels were closely correlated with worse outcomes of patients who had stroke. ${ }^{10}$ Neutrophils play crucial roles in the innate cellular immune system. ${ }^{11}$ Previous studies suggested that early higher neutrophil counts were correlated with increased stroke severity. ${ }^{12}$ Nevertheless, to our knowledge, no previous study has focused on the NPAR. In this study, we hypothesized that NPAR is a novel biomarker of inflammation associated with all-cause Mortality in patients with stroke.

Therefore, in this study, the data from the latest MIMIC-III (Multiparameter Intelligent Monitoring in Intensive Care) database 18 were used to investigate the association between NPAR and outcomes of stroke. Various potential confounders were adjusted.

\section{Methods}

\section{Study Population}

The protocol was approved by Massachusetts Institute of Technology and the Institutional Review Boards. The vital signs, medications, demographic information and other essential data of the patients admitted to intensive care unit (53,423 distinct admissions) from 2001 to 2012 in the Beth Israel Deaconess Medical Center (BIDMC, Boston) were collected, which were downloaded from MIMIC-III version 1.4. ${ }^{13}$ Investigators had learned the "Data or Specimens Only Research" online course. All data accessed complies with relevant data protection and privacy regulations.

\section{Population Selection Criteria}

We enrolled stroke patients (over 16 years) first admitted to the hospital with hospital stay over one day. And the patients were excluded if they stayed in ICU less than 48 hours, or had no data on the NPAR within the first 24 hours of admission.

\section{Study Variables and Outcomes}

The data upon admission were recorded, including vital signs, laboratory parameters, demographics, and comorbidities, etc. Comorbidities included malignancy, AF (atrial fibrillation), liver disease, pneumonia, renal disease, respiratory failure, CAD (coronary artery disease), and CHF (congestive heart failure). Laboratory parameters were WBC (white blood cells), BUN (blood urea nitrogen), APTT (activated partial thromboplastin time), PT (prothrombin time), potassium, anion gap, hemoglobin, creatinine, glucose, lactate, chloride, bicarbonate, sodium, platelet, hematocrit, albumin, bilirubin, INR (international normalized ratio), and the percentage of neutrophil. SAPSII (simplified acute physiology scores II) and APSIII (acute physiology score III) scores were also calculated. Furthermore, MBP (mean blood pressure), heart rate, DBP (diastolic blood pressure), respiratory rate, SBP (systolic blood pressure), SPO2, temperature, ethnicity, gender, and age were also recorded.

The primary outcome was 30-day mortality rate in our study, and the secondary outcomes included 90-day and 1 -year mortality rates. The patients were followed up for at least one year since admission. The date of death was based on Social Security Death Index records.

\section{Statistical Analysis}

Three subgroups were set up based on the NPAR. Continuous variables were expressed as mean \pm SD (standard deviation), and categorical data as percentage or frequency. $\chi^{2}$ tests and Kruskal-Wallis $H$-test were employed to compare differences in baseline features between subgroups of NPAR for categoric variables and continuous variables, respectively. And then, the COX regression was used to assess the association between NPAR and outcomes of stroke patients. There was no covariate adjustment in Model 1. Age, ethnicity and gender were adjusted in Model 2. Confounders, eg, age, gender, ethnicity, sodium, chloride, CHF (yes/no), CAD (yes/no), AF (yes/no), renal disease (yes/no), liver disease (yes/no), and COPD (yes/no) were adjusted in Model 3. The relationship between 30-day all-cause mortality and NPAR was analyzed to investigate if NPAR effects differed among different subgroups.

$\mathrm{R}$ (Version 3.6.1) was used in all the analyses. $\mathrm{P}$ values were two-sided. $\mathrm{P}$ values $<0.05$ was considered statistically significant.

\section{Results}

\section{Subject Characteristics}

In our study, 940 participants ( $\geq 16$ years old) were included. There were 516 males and 424 females. The average age was $68.8 \pm 14.7$ years, and the average NPAR value $23.5 \pm 4.2$. 
Characteristics of the samples related to NPAR are listed in Table 1. The subjects were categorized into 3 groups based on NPAR values: group A: NPAR $<20.5$ (313); group B: NPAR 20.5-25.0 (313); and group C: NPAR $>25.0$ (314). Compared with group A, participants in groups with higher NPAR $(>25.0)$ showed lower SBP, bicarbonate, MBP, hematocrit, hemoglobin, and DBP, and had higher levels of creatinine, BUN, potassium, chloride, heart rate, respiratory rate, PT, APTT, INR, and increased the proportion of CHF, renal disease, pneumonia, respiratory failure, and mortality. In addition, tertiles were not significantly related to age, ethnicity and gender in our study population.

\section{Association Between NAPR and Outcomes of Stroke Patients}

Different models were used to assess independent effects of NAPR and the outcomes of stroke patients after other potential confounders were adjusted. Effect sizes (HR) and 95\% CIs are shown in Table 2. For 30day all-cause mortality, in an unadjusted model, the HR (95\% CIs) in group B and C were $1.17(0.85,1.63)$ and $1.55(1.13,2.11)$, respectively, in comparison with group A. This association was significant after adjusting for age, gender, ethnicity, sodium, chloride, $\mathrm{CHF}, \mathrm{CAD}, \mathrm{AF}$, renal disease, liver disease, $\mathrm{COPD}(\mathrm{HR}=1.45,95 \% \mathrm{CI}$ : $1.05,2.00)$. The trend was also statistically significant $(P=0.0196)$. For 90 -day all-cause mortality and 365 day all-cause mortality, a similar relationship was also observed.

\section{Subgroup Analysis}

Subgroup analysis was performed to investigate the relationship between 30-day all-cause mortality and NPAR (Table 3). No interaction was observed in most strata $(\mathrm{P}=0.0650-0.9761)$. Only patients with WBC $<13.1 * 10^{9} / 1$ had higher risks of all-cause mortality for high NPAR.

\section{Discussion}

To our knowledge, we first explored the correlations between NPAR and the mortality of stroke patients in the short term and long term. We found that higher NPAR was closely associated with increased all-cause mortality of stroke patients in the short or long term. Compared with the complex scoring systems, NPAR is easy to access and can help clinicians quickly make clinical strategies in time.

Strokes occur due to cerebral vascular occlusion or hemorrhage resulting in deprivation of oxygen and nutrients, causing a local inflammatory immune response. ${ }^{14}$ This results in alteration of the systemic inflammatory response via the sympathetic pathway and the hypothalamus-pituitary-adrenal axis (poststroke immunosuppression) resulting in neutrophil demargination and stimulation of growth factors. ${ }^{15,16}$ A positive correlation was observed within acute ischemic stroke patients, between total WBC-neutrophil counts proceeding 3 days of symptom onset severity and infarct volume. $^{17,18}$

It has been suggested by prior studies, that the relationship between hypoproteinemia and stroke-related mortality, heart attack and fractures of the hip illustrate poorer disease prognosis with lower albumin values. ${ }^{10,19,20}$ According to previous studies, albumin may exert neuroprotective function via its antiinflammatory activity, antioxidant characteristics, inhibiting endothelial apoptosis, and regulating microvascular permeability. ${ }^{21-23}$ Another theory is that low albumin in time of acute stroke could illustrate the role albumin's concentration plays during inflammation states as a negative acute phase reactant. ${ }^{24}$ Negative regulation of the albumin synthesis may be controlled via interleukin (IL-6 and tumor necrosis factor, which are seen in increased concentrations during states of acute inflammation, such as acute stroke. ${ }^{25}$ In this serum albumin repression a possible detrimental effect may interfere with albumin's antioxidative and endothelial effects. ${ }^{26}$ Based on our results, the novel biomarker that is NPAR can be used to significantly predict stroke prognosis via neutrophil percentage and albumin levels.

Our study had some limitations. First, selection bias existed since this was a single-center retrospective study. Second, NPAR was only recorded when patients were admitted into the ICU and subsequent changes were not assessed. Third, other known and unknown factors still remain although we have made the best effort to control bias. In addition, the stroke severity score (National 
Table I Characteristics of the Study Patients According to Neutrophil Percentage-to-Albumin Ratios

\begin{tabular}{|c|c|c|c|c|}
\hline \multirow[t]{2}{*}{ Characteristics } & \multicolumn{4}{|c|}{ Neutrophil Percentage-to-Albumin Ratios } \\
\hline & $<20.5(n=313)$ & $\geq 20.5,<25.0(n=313)$ & $\geq 25.0(n=314)$ & $P$ value \\
\hline Age, years & $66.5 \pm 14.8$ & $67.2 \pm 15.0$ & $67.0 \pm 14.2$ & 0.839 \\
\hline Gender, n (\%) & & & & 0.893 \\
\hline Female & $139(44.4)$ & $140(44.7)$ & $145(46.2)$ & \\
\hline Male & $174(55.6)$ & $173(55.3)$ & $169(53.8)$ & \\
\hline Ethnicity, n (\%) & & & & 0.328 \\
\hline White & $221(70.6)$ & $224(71.6)$ & $224(71.3)$ & \\
\hline Black & $34(10.9)$ & $20(6.4)$ & $28(8.9)$ & \\
\hline Other & $58(18.5)$ & $69(22.0)$ & $62(19.7)$ & \\
\hline NPAR & $16.7 \pm 4.2$ & $22.6 \pm 1.3$ & $31.3 \pm 7.1$ & $<0.001$ \\
\hline $\mathrm{SBP}, \mathrm{mmHg}$ & $130.5 \pm 17.8$ & $129.4 \pm 17.5$ & $121.4 \pm 17.7$ & $<0.001$ \\
\hline $\mathrm{DBP}, \mathrm{mmHg}$ & $64.5 \pm 11.1$ & $64.8 \pm 11.7$ & $61.5 \pm 11.0$ & $<0.001$ \\
\hline MBP, mmHg & $83.5 \pm 11.4$ & $83.7 \pm 12.0$ & $79.3 \pm 11.3$ & $<0.001$ \\
\hline Heart rate, beats/minute & $80.4 \pm 16.2$ & $81.8 \pm 14.5$ & $87.6 \pm 17.4$ & $<0.001$ \\
\hline Respiratory rate, beats/minute & $18.4 \pm 3.5$ & $18.4 \pm 3.4$ & $20.3 \pm 4.7$ & $<0.001$ \\
\hline Temperature, ${ }^{\circ} \mathrm{C}$ & $36.9 \pm 0.6$ & $37.0 \pm 0.6$ & $36.9 \pm 0.8$ & 0.861 \\
\hline $\mathrm{SPO} 2, \%$ & $97.5 \pm 1.9$ & $97.8 \pm 2.1$ & $97.5 \pm 2.6$ & 0.307 \\
\hline \multicolumn{5}{|l|}{ Comorbidities, n (\%) } \\
\hline Congestive heart failure & $28(8.9)$ & $26(8.3)$ & $52(16.6)$ & 0.001 \\
\hline Coronary artery disease & $43(13.7)$ & $64(20.4)$ & $56(17.8)$ & 0.082 \\
\hline Atrial fibrillation & $84(26.8)$ & $90(28.8)$ & $108(34.4)$ & 0.100 \\
\hline Renal disease & $27(8.6)$ & $35(11.2)$ & $64(20.4)$ & $<0.001$ \\
\hline Liver disease & $13(4.2)$ & $10(3.2)$ & $22(7.0)$ & 0.067 \\
\hline Pneumonia & $72(23.0)$ & $84(26.8)$ & $126(40.1)$ & $<0.001$ \\
\hline Malignancy & $52(16.6)$ & $38(12.1)$ & $48(15.3)$ & 0.267 \\
\hline Respiratory failure & $79(25.2)$ & $101(32.3)$ & $184(58.6)$ & $<0.001$ \\
\hline COPD & I (0.3) & $3(1.0)$ & $4(1.3)$ & 0.416 \\
\hline \multicolumn{5}{|l|}{ Laboratory parameters } \\
\hline Neutrophil percentage, \% & $66.9 \pm 18.9$ & $83.3 \pm 7.5$ & $85.4 \pm 7.2$ & $<0.001$ \\
\hline Albumin, g/dl & $4.0 \pm 0.6$ & $3.7 \pm 0.4$ & $2.8 \pm 0.5$ & $<0.001$ \\
\hline Bicarbonate, $\mathrm{mg} / \mathrm{dl}$ & $25.8 \pm 3.7$ & $25.3 \pm 3.5$ & $24.3 \pm 4.8$ & $<0.001$ \\
\hline Anion gap, mmol/l & $16.8 \pm 3.8$ & $16.9 \pm 3.8$ & $17.5 \pm 5.1$ & 0.110 \\
\hline Creatinine, $\mathrm{mEq} / \mathrm{l}$ & $1.4 \pm 2.5$ & $1.5 \pm 1.8$ & $2.0 \pm 1.7$ & $<0.001$ \\
\hline Chloride, $\mathrm{mmol} / \mathrm{l}$ & $106.6 \pm 6.1$ & $107.2 \pm 6.7$ & $108.8 \pm 7.4$ & $<0.001$ \\
\hline Glucose, mg/dl & $178.5 \pm 87.1$ & $192.5 \pm 81.9$ & $196.7 \pm 94.2$ & 0.025 \\
\hline Hematocrit, \% & $38.3 \pm 6.1$ & $38.3 \pm 5.3$ & $35.2 \pm 6.1$ & $<0.001$ \\
\hline Hemoglobin, g/dl & $13.0 \pm 2.1$ & $13.0 \pm 1.9$ & $11.7 \pm 2.2$ & $<0.001$ \\
\hline Platelet, $10^{9} / 1$ & $246.3 \pm 112.6$ & $258.6 \pm 105.3$ & $250.6 \pm 144.0$ & 0.446 \\
\hline Sodium, mmol/l & $141.5 \pm 5.3$ & $14 \mid .6 \pm 5.4$ & $141.5 \pm 5.6$ & 0.972 \\
\hline Potassium, mmol/l & $4.4 \pm 0.8$ & $4.4 \pm 0.8$ & $4.7 \pm 0.9$ & $<0.001$ \\
\hline BUN, mg/dl & $24.1 \pm 19.1$ & $24.4 \pm 16.7$ & $38.5 \pm 29.3$ & $<0.001$ \\
\hline WBC, $10^{9} / I$ & $13.9 \pm 21.5$ & $14.0 \pm 6.1$ & $15.9 \pm 8.9$ & 0.109 \\
\hline PT, second & $15.9 \pm 9.2$ & $15.8 \pm 9.1$ & $20.0 \pm 14.4$ & $<0.001$ \\
\hline APTT, second & $35.4 \pm 21.2$ & $37.1 \pm 26.4$ & $50.2 \pm 36.5$ & $<0.001$ \\
\hline INR & $1.5 \pm 1.2$ & $1.5 \pm 1.4$ & $2.0 \pm 1.9$ & $<0.001$ \\
\hline
\end{tabular}

(Continued) 
Table I (Continued).

\begin{tabular}{|c|c|c|c|c|}
\hline \multirow{2}{*}{ Characteristics } & \multicolumn{4}{|c|}{ Neutrophil Percentage-to-Albumin Ratios } \\
\hline & $<20.5(n=313)$ & $\geq 20.5,<25.0(n=313)$ & $\geq 25.0(n=314)$ & $P$ value \\
\hline \multicolumn{5}{|l|}{ Scoring systems } \\
\hline APSIII & $42.9 \pm 19.9$ & $43.3 \pm 20.0$ & $57.9 \pm 24.8$ & $<0.001$ \\
\hline SAPSII & $35.9 \pm 13.8$ & $38.4 \pm 13.1$ & $46.4 \pm 14.6$ & $<0.001$ \\
\hline 30-day mortality, n (\%) & $67(21.4)$ & $77(24.6)$ & $100(31.8)$ & 0.009 \\
\hline 90-day mortality, n (\%) & $78(24.9)$ & $92(29.4)$ & $125(39.8)$ & $<0.001$ \\
\hline 365-day mortality, n (\%) & $97(31.0)$ & $107(34.2)$ & $145(46.2)$ & $<0.001$ \\
\hline
\end{tabular}

Abbreviations: NPAR, neutrophil percentage-to-albumin ratio; SBP, systolic blood pressure; DBP, diastolic blood pressure; MBP, mean blood pressure; COPD, chronic obstructive pulmonary disease; BUN, blood urea nitrogen; WBC, white blood cell; PT, prothrombin time; APTT, activated partial thromboplastin time; INR, international normalized ratio; APSIII, acute physiology score III; SAPSII, simplified acute physiology score II.

Table 2 HRs (95\% Cls) for All-Cause Mortality Across Groups of Neutrophil Percentage-to-Albumin Ratios

\begin{tabular}{|c|c|c|c|c|c|c|}
\hline \multirow[t]{2}{*}{ NAR } & \multicolumn{2}{|c|}{ Non-Adjusted } & \multicolumn{2}{|c|}{ Model I } & \multicolumn{2}{|c|}{ Model II } \\
\hline & HR (95\% Cls) & $P$ value & HR (95\% Cls) & $P$ value & HR (95\% Cls) & $P$ value \\
\hline \multicolumn{7}{|c|}{ 30-day all-cause mortality } \\
\hline \multicolumn{7}{|l|}{ Tertiles } \\
\hline$<20.5$ & I.0 (ref) & & I.0 (ref) & & 1.0 (ref) & \\
\hline$\geq 20.5,<25.0$ & $1.17(0.85,1.63)$ & 0.3389 & I.II $(0.80,1.55)$ & 0.5175 & $1.10(0.79,1.54)$ & 0.5670 \\
\hline$\geq 25.0$ & $1.55(1.13,2.11)$ & 0.0058 & $1.52(\mathrm{I} .1 \mathrm{I}, 2.07)$ & 0.0083 & $1.45(1.05,2.00)$ & 0.0254 \\
\hline$P$ trend & 0.0044 & & 0.0054 & & 0.0196 & \\
\hline \multicolumn{7}{|c|}{ 90-day all-cause mortality } \\
\hline \multicolumn{7}{|l|}{ Tertiles } \\
\hline$<20.5$ & I.0 (ref) & & I.0 (ref) & & I.0 (ref) & \\
\hline$\geq 20.5,<25.0$ & $1.21(0.90,1.64)$ & 0.2102 & $1.16(0.86,1.57)$ & 0.3421 & $1.15(0.85,1.56)$ & 0.3719 \\
\hline$\geq 25.0$ & I.7I $(1.29,2.26)$ & 0.0002 & $1.67(1.26,2.22)$ & 0.0004 & $1.60(1.19,2.15)$ & 0.0020 \\
\hline$P$ trend & 0.0001 & & 0.0002 & & 0.0013 & \\
\hline \multicolumn{7}{|c|}{ 365-day all-cause mortality } \\
\hline \multicolumn{7}{|l|}{ Tertiles } \\
\hline$<20.5$ & I.0 (ref) & & 1.0 (ref) & & 1.0 (ref) & \\
\hline$\geq 20.5,<25.0$ & $1.14(0.87,1.50)$ & 0.3539 & $1.08(0.82,1.42)$ & 0.5774 & $\mathrm{I} .08(0.8 \mathrm{I}, \mathrm{I} .42)$ & 0.6105 \\
\hline$\geq 25.0$ & $1.63(1.26,2.11)$ & 0.0002 & $1.60(1.24,2.07)$ & 0.0003 & $1.50(1.15,1.97)$ & 0.0030 \\
\hline$P$ trend & $<0.0001$ & & 0.0001 & & 0.0017 & \\
\hline
\end{tabular}

Notes: Models were derived from Cox proportional hazards regression models. Non-adjusted model adjust for: none. Adjust I model adjust for: age, ethnicity and gender. Adjust II model adjust for: age, gender, ethnicity, sodium, chloride, congestive heart failure, coronary artery disease, atrial fibrillation, renal disease, liver disease, chronic obstructive pulmonary disease.

Abbreviations: $\mathrm{HR}$, hazard ratio; $\mathrm{Cl}$, confidence interval.

Institute of Health stroke scale) was not included in our study, which might have some significance. Furthermore, there were inaccurate data in the database. Thus, the findings still require verification by multi-center prospective studies.

\section{Conclusions}

We demonstrated that higher NPAR was closely associated with increased all-cause mortality in stroke patients. Nevertheless, these findings need to be confirmed by large prospective multicenter studies. 
Table 3 Subgroup Analysis of the Associations Between the Neutrophil Percentage-to-Albumin Ratios and 30-Day All-Cause Mortality

\begin{tabular}{|c|c|c|c|c|c|}
\hline & \multirow[t]{2}{*}{ No. of Patients } & \multicolumn{3}{|c|}{ Neutrophil Percentage-to-Albumin Ratios } & \multirow[t]{2}{*}{$P$ for Interaction } \\
\hline & & $<20.5$ & $\geq 20.5,<25.0$ & $\geq \mathbf{2 5 . 0}$ & \\
\hline Age, years & & & & & 0.2351 \\
\hline$<69.2$ & 470 & $\mathrm{I} .0$ (ref) & I.7I $(0.98,2.97)$ & $2.15(1.28,3.60)$ & \\
\hline$\geq 69.2$ & 470 & $\mathrm{I} .0$ (ref) & $0.84(0.56,1.27)$ & $1.19(0.81,1.76)$ & \\
\hline Gender & & & & & 0.8199 \\
\hline Female & 424 & I.0 (ref) & $1.26(0.80,2.00)$ & $1.69(1.10,2.61)$ & \\
\hline Male & 516 & I.0 (ref) & $1.09(0.69,1.74)$ & $1.39(0.89,2.17)$ & \\
\hline Ethnicity & & & & & 0.1416 \\
\hline White & 669 & I.0 (ref) & $1.12(0.76,1.65)$ & $1.47(1.02,2.13)$ & \\
\hline Black & 82 & $\mathrm{I} .0$ (ref) & $4.60(0.89,23.72)$ & $4.58(0.95,22.07)$ & \\
\hline Other & 189 & I.0 (ref) & $0.90(0.47,1.72)$ & $1.34(0.72,2.5 \mathrm{I})$ & \\
\hline $\mathrm{SBP}, \mathrm{mmHg}$ & & & & & 0.5523 \\
\hline$<127$ & 469 & 1.0 (ref) & $1.13(0.67,1.88)$ & $1.63(1.04,2.56)$ & \\
\hline$\geq 127$ & 470 & I.0 (ref) & $1.21(0.79,1.86)$ & $1.47(0.94,2.32)$ & \\
\hline $\mathrm{DBP}, \mathrm{mmHg}$ & & & & & 0.1894 \\
\hline$<63$ & 469 & I.0 (ref) & $0.78(0.48,1.27)$ & $1.30(0.85,1.98)$ & \\
\hline$\geq 63$ & 470 & 1.0 (ref) & $\mathrm{I} .66(\mathrm{I} .05,2.6 \mathrm{I})$ & $1.85(1.17,2.93)$ & \\
\hline $\mathrm{MBP}, \mathrm{mmHg}$ & & & & & 0.3216 \\
\hline$<82$ & 469 & I.0 (ref) & $0.89(0.54,1.47)$ & $1.40(0.91,2.15)$ & \\
\hline$\geq 82$ & 470 & I.0 (ref) & $1.45(0.93,2.24)$ & $1.72(1.09,2.70)$ & \\
\hline Respiratory rate, beats/minute & & & & & 0.6852 \\
\hline$<18$ & 469 & 1.0 (ref) & $1.34(0.84,2.12)$ & $1.74(1.08,2.80)$ & \\
\hline$\geq 18$ & 469 & I.0 (ref) & $1.00(0.63,1.59)$ & $1.30(0.86,1.96)$ & \\
\hline Temperature, ${ }^{\circ} \mathrm{C}$ & & & & & 0.2201 \\
\hline$<36.9$ & 466 & I.0 (ref) & $\mathrm{I} .57(0.98,2.5 \mathrm{I})$ & $1.69(1.08,2.66)$ & \\
\hline$\geq 36.9$ & 467 & I.0 (ref) & $0.89(0.56,1.40)$ & $1.45(0.94,2.21)$ & \\
\hline SPO2, \% & & & & & 0.3479 \\
\hline$<98$ & 469 & I.0 (ref) & $1.12(0.66,1.88)$ & $1.67(1.04,2.70)$ & \\
\hline$\geq 98$ & 470 & I.0 (ref) & $1.14(0.75,1.74)$ & $1.38(0.92,2.07)$ & \\
\hline Sodium, $\mathrm{mmol} / \mathrm{l}$ & & & & & 0.6897 \\
\hline$<140$ & 423 & 1.0 (ref) & $1.42(0.85,2.38)$ & $1.83(1.11,3.01)$ & \\
\hline$\geq 140$ & 514 & 1.0 (ref) & $1.02(0.66,1.57)$ & $1.37(0.92,2.05)$ & \\
\hline Potassium, $\mathrm{mmol} / \mathrm{l}$ & & & & & 0.4276 \\
\hline$<4.3$ & 427 & 1.0 (ref) & $1.24(0.76,2.02)$ & $2.09(1.30,3.37)$ & \\
\hline$\geq 4.3$ & 510 & I.0 (ref) & $1.13(0.73,1.77)$ & $1.26(0.84,1.90)$ & \\
\hline Chloride, $\mathrm{mmol} / \mathrm{l}$ & & & & & 0.5696 \\
\hline$<107$ & 439 & I.0 (ref) & $1.05(0.65,1.69)$ & $1.84(1.17,2.89)$ & \\
\hline$\geq 107$ & 498 & I.0 (ref) & $1.29(0.8 \mathrm{I}, 2.04)$ & $1.39(0.90,2.15)$ & \\
\hline WBC, $10^{9} / 1$ & & & & & 0.0215 \\
\hline$<|3|$. & 462 & I.0 (ref) & $1.57(0.94,2.64)$ & $2.42(1.47,3.98)$ & \\
\hline$\geq 13.1$ & 475 & I.0 (ref) & $0.85(0.55,1.30)$ & $1.00(0.67,1.48)$ & \\
\hline
\end{tabular}

(Continued) 
Table 3 (Continued).

\begin{tabular}{|c|c|c|c|c|c|}
\hline & \multirow[t]{2}{*}{ No. of Patients } & \multicolumn{3}{|c|}{ Neutrophil Percentage-to-Albumin Ratios } & \multirow[t]{2}{*}{$P$ for Interaction } \\
\hline & & $<20.5$ & $\geq 20.5,<25.0$ & $\geq \mathbf{2 5 . 0}$ & \\
\hline Platelet, $10^{9} / /$ & & & & & 0.4410 \\
\hline$<239$ & 463 & $\mathrm{I} .0$ (ref) & $1.09(0.68,1.75)$ & $1.59(1.04,2.43)$ & \\
\hline$\geq 239$ & 474 & $\mathrm{I} .0$ (ref) & $1.28(0.80,2.02)$ & $1.52(0.96,2.40)$ & \\
\hline Hematocrit, \% & & & & & 0.3502 \\
\hline$<37.4$ & 463 & $\mathrm{I} .0$ (ref) & $0.98(0.60,1.62)$ & $1.37(0.89,2.11)$ & \\
\hline$\geq 37.4$ & 474 & 1.0 (ref) & $1.35(0.87,2.10)$ & $1.74(1.09,2.77)$ & \\
\hline Hemoglobin, g/dl & & & & & 0.9761 \\
\hline$<12.6$ & 454 & $\mathrm{I} .0$ (ref) & $1.20(0.73,1.98)$ & $1.49(0.96,2.32)$ & \\
\hline$\geq 12.6$ & 483 & 1.0 (ref) & $1.16(0.75,1.80)$ & $1.61(1.02,2.55)$ & \\
\hline Creatinine, $\mathrm{mEq} / \mathrm{l}$ & & & & & 0.0650 \\
\hline$<$ I.I & 446 & 1.0 (ref) & I.46 $(0.87,2.44)$ & $2.17(1.29,3.65)$ & \\
\hline$\geq \mathrm{I} . \mathrm{I}$ & 491 & 1.0 (ref) & $1.00(0.65,1.55)$ & $1.15(0.78,1.69)$ & \\
\hline BUN, mg/dl & & & & & 0.1011 \\
\hline$<24$ & 440 & $\mathrm{I} .0$ (ref) & $1.59(0.98,2.59)$ & $1.90(1.09,3.32)$ & \\
\hline$\geq 24$ & 497 & 1.0 (ref) & $0.87(0.56,1.37)$ & $1.14(0.78,1.68)$ & \\
\hline Anion gap, mmol/l & & & & & 0.5589 \\
\hline$<16$ & 362 & $\mathrm{I} .0$ (ref) & $1.02(0.53,1.94)$ & $\mathrm{I} .35(0.74,2.47)$ & \\
\hline$\geq 16$ & 574 & I.0 (ref) & $\mathrm{I} .23(0.84, \mathrm{I} .8 \mathrm{I})$ & $1.64(1.14,2.36)$ & \\
\hline Bicarbonate, mg/dl & & & & & 0.5392 \\
\hline$<25$ & 398 & $\mathrm{I} .0$ (ref) & $1.00(0.62,1.63)$ & $1.17(0.74,1.84)$ & \\
\hline$\geq 25$ & 538 & $\mathrm{I} .0$ (ref) & $1.26(0.80,1.98)$ & $1.83(1.19,2.82)$ & \\
\hline Glucose, $\mathrm{mg} / \mathrm{dl}$ & & & & & 0.1462 \\
\hline$<164$ & 467 & I.0 (ref) & $0.94(0.53,1.65)$ & $1.80(1.10,2.95)$ & \\
\hline$\geq 164$ & 470 & I.0 (ref) & $1.16(0.76,1.75)$ & $1.25(0.84,1.87)$ & \\
\hline PT, second & & & & & 0.5228 \\
\hline$<14$ & 456 & 1.0 (ref) & $1.06(0.65,1.72)$ & $1.24(0.72,2.15)$ & \\
\hline$\geq 14$ & 471 & 1.0 (ref) & $1.31(0.83,2.05)$ & $1.49(1.00,2.22)$ & \\
\hline APTT, second & & & & & 0.5421 \\
\hline$<30$ & 461 & $\mathrm{I} .0$ (ref) & $0.90(0.56,1.44)$ & $1.09(0.64,1.85)$ & \\
\hline$\geq 30$ & 464 & $\mathrm{I} .0$ (ref) & $1.62(1.01,2.57)$ & $1.77(1.17,2.68)$ & \\
\hline INR & & & & & 0.2899 \\
\hline$<1.3$ & 446 & $\mathrm{I} .0$ (ref) & $0.89(0.55,1.44)$ & $1.11(0.64,1.91)$ & \\
\hline$\geq 1.3$ & 481 & 1.0 (ref) & $1.51(0.96,2.38)$ & $1.65(1.10,2.50)$ & \\
\hline $\mathrm{CHF}$ & & & & & 0.1365 \\
\hline No & 834 & $\mathrm{I} .0$ (ref) & $1.27(0.91,1.79)$ & $\mathrm{I} .73(\mathrm{I} .25,2.4 \mathrm{I})$ & \\
\hline Yes & 106 & 1.0 (ref) & $0.38(0.10,1.45)$ & $0.7 \mathrm{I}(0.29, \mathrm{I} .77)$ & \\
\hline AFIB & & & & & 0.9222 \\
\hline No & 658 & $\mathrm{I} .0$ (ref) & I.22 (0.82, I.82) & $1.54(1.05,2.26)$ & \\
\hline Yes & 282 & 1.0 (ref) & $1.07(0.60,1.89)$ & $\mathrm{I} .49(0.88,2.5 \mathrm{I})$ & \\
\hline CAD & & & & & 0.0806 \\
\hline No & 777 & $\mathrm{I} .0$ (ref) & $1.37(0.96,1.95)$ & $1.58(1.12,2.22)$ & \\
\hline Yes & 163 & $\mathrm{I} .0$ (ref) & $0.53(0.23,1.23)$ & $1.35(0.66,2.76)$ & \\
\hline
\end{tabular}

(Continued) 
Table 3 (Continued).

\begin{tabular}{|c|c|c|c|c|c|}
\hline & \multirow[t]{2}{*}{ No. of Patients } & \multicolumn{3}{|c|}{ Neutrophil Percentage-to-Albumin Ratios } & \multirow[t]{2}{*}{$P$ for Interaction } \\
\hline & & $<20.5$ & $\geq 20.5,<25.0$ & $\geq \mathbf{2 5 . 0}$ & \\
\hline Malignancy & & & & & 0.5813 \\
\hline No & 802 & 1.0 (ref) & $1.24(0.87,1.76)$ & $1.54(1.10,2.17)$ & \\
\hline Yes & 138 & 1.0 (ref) & $0.78(0.31,1.99)$ & $1.56(0.74,3.30)$ & \\
\hline Liver disease & & & & & 0.0806 \\
\hline No & 895 & 1.0 (ref) & $\mathrm{I} .10(0.78, \mathrm{I} .54)$ & $1.52(1.10,2.09)$ & \\
\hline Yes & 45 & 1.0 (ref) & $4.28(1.10,16.67)$ & $1.81(0.49,6.70)$ & \\
\hline Renal disease & & & & & 0.1191 \\
\hline No & 814 & 1.0 (ref) & $1.30(0.91,1.85)$ & $1.69(1.20,2.39)$ & \\
\hline Yes & 126 & 1.0 (ref) & $0.55(0.23,1.3 \mathrm{I})$ & $0.74(0.37,1.50)$ & \\
\hline Respiratory failure & & & & & 0.7417 \\
\hline No & 576 & 1.0 (ref) & $\mathrm{I} .07(0.7 \mathrm{I}, \mathrm{I} .6 \mathrm{I})$ & $1.46(0.95,2.25)$ & \\
\hline Yes & 364 & $\mathrm{I} .0$ (ref) & $\mathrm{I} .30(0.74,2.27)$ & $1.42(0.86,2.35)$ & \\
\hline Pneumonia & & & & & 0.0696 \\
\hline No & 658 & 1.0 (ref) & $\mathrm{I} .42(0.96,2.09)$ & I.91 (I.31, 2.80) & \\
\hline Yes & 282 & 1.0 (ref) & $0.68(0.36,1.27)$ & $0.93(0.55,1.59)$ & \\
\hline COPD & & & & & 0.3686 \\
\hline No & 932 & 1.0 (ref) & I.I8 $(0.85,1.65)$ & $1.55(1.13,2.12)$ & \\
\hline Yes & 8 & 1.0 (ref) & $0.37(0.02,5.97)$ & $0.77(0.07,8.58)$ & \\
\hline SAPSII & & & & & 0.3904 \\
\hline$<38$ & 437 & 1.0 (ref) & $1.63(0.91,2.91)$ & $\mathrm{I} .32(0.65,2.7 \mathrm{I})$ & \\
\hline$\geq 38$ & 503 & 1.0 (ref) & $0.85(0.57,1.26)$ & $0.99(0.70,1.42)$ & \\
\hline APSIII & & & & & 0.7695 \\
\hline$<43$ & 459 & 1.0 (ref) & $1.39(0.81,2.37)$ & $1.80(1.01,3.24)$ & \\
\hline$\geq 43$ & 481 & 1.0 (ref) & $1.08(0.71,1.63)$ & $1.09(0.75,1.58)$ & \\
\hline
\end{tabular}

Abbreviations: SBP, systolic blood pressure; DBP, diastolic blood pressure; MBP, mean blood pressure; WBC, white blood cell; BUN, blood urea nitrogen; PT, prothrombin time; APTT, activated partial thromboplastin time; INR, international normalized ratio; CHF, congestive heart failure; AFIB, atrial fibrillation; CAD, coronary artery disease; COPD, chronic obstructive pulmonary disease; SAPSII, simplified acute physiology scorell.

\section{Acknowledgment}

Thanks for the support of Wenzhou Municipal Scientific and Technological Program Projects (no. Y20190133) and Research Incubation Projects of the First Affiliated Hospital of Wenzhou Medical University (no. FHY2019017).

\section{Disclosure}

The authors report no conflicts of interest in this work.

\section{References}

1. Liu L, Wang D, Wong KS, Wang Y. Stroke and stroke care in China: huge burden, significant workload, and a national priority. Stroke. 2011;42(12):3651-3654. doi:10.1161/STROKEAHA.111.635755

2. Zhou M, Wang H, Zeng X, et al. Mortality, morbidity, and risk factors in China and its provinces, 1990-2017: a systematic analysis for the Global Burden of Disease Study 2017. Lancet. 2019;394 (10204):1145-1158. doi:10.1016/S0140-6736(19)30427-1
3. Powers WJ, Rabinstein AA, Ackerson T, et al. Guidelines for the early management of patients with acute ischemic stroke: 2019 update to the 2018 Guidelines for the Early Management of Acute Ischemic Stroke: a Guideline for Healthcare Professionals From the American Heart Association/American Stroke Association. Stroke. 2019;50(12):e344-e418.

4. Wang Y, Zhao X, Jiang Y, et al. Prevalence, knowledge, and treatment of transient ischemic attacks in China. Neurology. 2015;84 (23):2354-2361. doi:10.1212/WNL.0000000000001665

5. McDermott M, Jacobs T, Morgenstern L. Critical care in acute ischemic stroke. Handb Clin Neurol. 2017;140:153-176.

6. Anrather J, Iadecola C. Inflammation and stroke: an overview. Neurotherapeutics. 2016;13(4):661-670. doi:10.1007/s13311-0160483-x

7. Chamorro Á, Dirnagl U, Urra X, Planas AM. Neuroprotection in acute stroke: targeting excitotoxicity, oxidative and nitrosative stress, and inflammation. Lancet Neurol. 2016;15(8):869-881. doi:10.1016/ S1474-4422(16)00114-9

8. Gong Y, Li D, Cheng B, Ying B, Wang B. Increased neutrophil percentage-to-albumin ratio is associated with all-cause mortality in patients with severe sepsis or septic shock. Epidemiol Infect. 2020;148:e87. doi:10.1017/S0950268820000771 
9. Sun T, Shen H, Guo Q, et al. Association between neutrophil percentage-to-albumin ratio and all-cause mortality in critically ill patients with coronary artery disease. Biomed Res Int. 2020;2020:8137576. doi:10.1155/2020/8137576

10. Famakin B, Weiss P, Hertzberg V, et al. Hypoalbuminemia predicts acute stroke mortality: Paul Coverdell Georgia Stroke Registry. J Stroke Cerebrovasc Dis. 2010;19(1):17-22. doi:10.1016/j. jstrokecerebrovasdis.2009.01.015

11. Liew PX, Kubes P. The Neutrophil's Role during health and disease. Physiol Rev. 2019;99(2):1223-1248. doi:10.1152/physrev.00012.2018

12. Kang L, Yu H, Yang X, et al. Neutrophil extracellular traps released by neutrophils impair revascularization and vascular remodeling after stroke. Nat Commun. 2020;11(1):2488. doi:10.1038/s41467-020-16191-y

13. Johnson AE, Pollard TJ, Shen L, et al. MIMIC-III, a freely accessible critical care database. Sci Data. 2016;3:160035. doi:10.1038/ sdata.2016.35

14. Shim R, Wong CH. Ischemia, immunosuppression and infectiontackling the predicaments of post-stroke complications. Int $\mathrm{J} \mathrm{Mol}$ Sci. 2016;17(1):64. doi:10.3390/ijms17010064

15. Hannawi Y, Hannawi B, Rao CP, Suarez JI, Bershad EM. Strokeassociated pneumonia: major advances and obstacles. Cerebrovasc Dis. 2013;35(5):430-443. doi:10.1159/000350199

16. Prass K, Meisel C, Höflich C, et al. Stroke-induced immunodeficiency promotes spontaneous bacterial infections and is mediated by sympathetic activation reversal by poststroke $\mathrm{T}$ helper cell type 1-like immunostimulation. $J$ Exp Med. 2003;198(5):725-736. doi:10.1084/jem.20021098

17. Westendorp WF, Vermeij JD, Zock E, et al. The Preventive Antibiotics in Stroke Study (PASS): a pragmatic randomised open-label masked endpoint clinical trial. Lancet. 2015;385 (9977):1519-1526. doi:10.1016/S0140-6736(14)62456-9

18. Nam KW, Kwon HM, Jeong HY, et al. High neutrophil to lymphocyte ratio is associated with white matter hyperintensity in a healthy population. J Neurol Sci. 2017;380:128-131. doi:10.1016/j.jns.2017.07.024
19. Plakht Y, Gilutz H, Shiyovich A. Decreased admission serum albumin level is an independent predictor of long-term mortality in hospital survivors of acute myocardial infarction. Soroka Acute Myocardial Infarction II (SAMI-II) project. Int $J$ Cardiol. 2016;219:20-24. doi:10.1016/j.ijcard.2016.05.067

20. Pioli G, Barone A, Giusti A, et al. Predictors of mortality after hip fracture: results from 1-year follow-up. Aging Clin Exp Res. 2006;18 (5):381-387. doi:10.1007/BF03324834

21. Yildirim T, Okutan O, Akpinar E, Yilmaz A, Isik HS. Neuroprotective effects of high-dose human albumin against traumatic spinal cord injury in rats. Bratisl Lek Listy. 2018;119(2):86-91.

22. Belayev L, Pinard E, Nallet H, et al. Albumin therapy of transient focal cerebral ischemia: in vivo analysis of dynamic microvascular responses. Stroke. 2002;33(4):1077-1084. doi:10.1161/hs0402.105555

23. Belayev L, Liu Y, Zhao W, Busto R, Ginsberg MD. Human albumin therapy of acute ischemic stroke: marked neuroprotective efficacy at moderate doses and with a broad therapeutic window. Stroke. 2001;32(2):553-560. doi:10.1161/01.STR.32.2.553

24. Ebersole JL, Cappelli D. Acute-phase reactants in infections and inflammatory diseases. Periodontol 2000. 2000;23:19-49. doi:10.1034/j.1600-0757.2000.2230103.x

25. Ramadori G, Van Damme J, Rieder H, Meyer Zum Büschenfelde KH. Interleukin 6, the third mediator of acute-phase reaction, modulates hepatic protein synthesis in human and mouse. Comparison with interleukin 1 beta and tumor necrosis factor-alpha. Eur J Immunol. 1988;18(8):1259-1264. doi:10.1002/eji.1830180817

26. Halliwell B. Albumin-an important extracellular antioxidant? Biochem Pharmacol. 1988;37(4):569-571. doi:10.1016/00062952(88)90126-8
International Journal of General Medicine

\section{Publish your work in this journal}

The International Journal of General Medicine is an international, peer-reviewed open-access journal that focuses on general and internal medicine, pathogenesis, epidemiology, diagnosis, monitoring and treatment protocols. The journal is characterized by the rapid reporting of reviews, original research and clinical studies

\section{Dovepress}

across all disease areas. The manuscript management system is completely online and includes a very quick and fair peer-review system, which is all easy to use. Visit http://www.dovepress.com/ testimonials.php to read real quotes from published authors. 